\title{
Les jeunes adultes à la découverte de nouvelles musiques : quel rôle pour les bibliothèques publiques?
}

Young Adults and the Quest for New Music: Where to Public

Libraries Fit In?

\section{Adultos jóvenes a la búsqueda de nuevas expresiones musicales: ¿Qué función desempeñan las bibliotecas públicas?}

Audrey Laplante

Volume 55, numéro 2, avril-juin 2009

URI : https://id.erudit.org/iderudit/1029089ar

DOI : https://doi.org/10.7202/1029089ar

Aller au sommaire du numéro

Éditeur(s)

Association pour l'avancement des sciences et des techniques de la documentation (ASTED)

ISSN

0315-2340 (imprimé)

2291-8949 (numérique)

Découvrir la revue

Citer cet article

Laplante, A. (2009). Les jeunes adultes à la découverte de nouvelles musiques : quel rôle pour les bibliothèques publiques ? Documentation et bibliothèques,

55(2), 57-65. https://doi.org/10.7202/1029089ar
Résumé de l'article

Cet article présente les résultats d'une étude sur les stratégies qu'utilisent les jeunes adultes pour découvrir de nouvelles musiques. Des entrevues ont été réalisées avec 15 adultes de la région de Montréal, âgés de 18 à 29 ans. L'analyse a révélé que, pour faire des découvertes, les participants comptent principalement sur : 1) leur cercle social ; 2) les ressources du Web ; et 3) les magasins de disques. Ils font preuve d'une confiance limitée envers les experts et considèrent la recherche de musique comme une activité de loisir. Des suggestions sont données quant à la façon de tirer profit de ces résultats pour améliorer les services offerts en bibliothèque publique.
Tous droits réservés ( $)$ Association pour l'avancement des sciences et des techniques de la documentation (ASTED), 2009
Ce document est protégé par la loi sur le droit d'auteur. L’utilisation des services d'Érudit (y compris la reproduction) est assujettie à sa politique d'utilisation que vous pouvez consulter en ligne.

https://apropos.erudit.org/fr/usagers/politique-dutilisation/ 


\title{
Les jeunes adultes à la découverte de nouvelles musiques : quel rôle pour les bibliothèques publiques?
}

\author{
AUDREY LAPLANTE \\ Professeure adjointe \\ École de bibliothéconomie et des sciences de l'information \\ Université de Montréal \\ audrey.laplante@umontreal.ca
}

\section{RÉSUMÉ | ABSTRACTS | RESUMEN}

Cet article présente les résultats d'une étude sur les stratégies qu'utilisent les jeunes adultes pour découvrir de nouvelles musiques. Des entrevues ont été réalisées avec 15 adultes de la région de Montréal, âgés de 18 à 29 ans. L'analyse a révélé que, pour faire des découvertes, les participants comptent principalement sur: 1) leur cercle social ; 2) les ressources du Web; et 3) les magasins de disques. Ils font preuve d'une confiance limitée envers les experts et considèrent la recherche de musique comme une activité de loisir. Des suggestions sont données quant à la façon de tirer profit de ces résultats pour améliorer les services offerts en bibliothèque publique.

\section{Young Adults and the Quest for New Music: Where to Public Libraries Fit In?}

This article presents the results of a study of the strategies used by young adults in their quest for new music. Interviews were conducted with 15 Montréal-area adults, aged 18 to 29 years. The analysis shows that, in order to find new music, the participants relied mainly on: 1) their social circle, 2) the resources available on the Web, and 3) record stores. Their confidence in the experts is limited and they consider the search for music as a leisure activity. With these results in hand, suggestions are made improve the services offered by the public library.

Adultos jóvenes a la búsqueda de nuevas expresiones musicales: ¿Qué función desempeñan las bibliotecas públicas?

Este artículo presenta los resultados de un estudio sobre las estrategias que utilizan los adultos jóvenes para descubrir nuevas expresiones musicales. Se han realizado entrevistas con 15 adultos de la región de Montreal, de entre 18 y 29 años de edad. El análisis ha revelado que, para realizar la búsqueda, los participantes cuentan principalmente con: 1) su círculo social; 2) los recursos de la Web; $y_{3}$ ) las tiendas de discos. Demuestran una confianza limitada frente a los expertos y consideran la búsqueda musical como una actividad recreativa. Se plantean sugerencias en cuanto a la manera de aprovechar estos resultados para mejorar los servicios que la biblioteca pública ofrece.
L A Musique enRegistrée a longtemps occupé une place timide dans nos bibliothèques publiques. Les obstacles étaient nombreux. Autrefois consignée sur des supports fragiles (que l'on pense au rouleau de cire, au disque vinyle ou à la cassette audio) qui requéraient au surplus un équipement spécifique tout aussi fragile, son acquisition représentait un investissement important en ressources matérielles, financières et humaines. Il s'agissait là d'un investissement que la plupart des bibliothèques publiques n'étaient pas disposées à faire, d'abord parce que les ressources étaient limitées, mais aussi parce qu'offrir un accès à la musique enregistrée n'était pas considéré comme un service essentiel.

Force est de constater que les choses ont changé. Lavènement du disque compact et une affirmation plus claire du rôle de divertissement et déveil aux arts des bibliothèques ont entraîné une augmentation phénoménale du nombre denregistrements musicaux dans leurs collections. De 1998 à 2005, alors que le nombre de livres dans les collections des bibliothèques publiques autonomes du Québec augmentait de $23 \%$, celui des disques compacts bondissait de près de $109 \%$ (Québec (Province) 2000 ; Québec (Province) 2008). Plus récemment, lapparition de services de diffusion de musique en continu, tels que Naxos Music Library ${ }^{1}$ ou Smithsonian Global Sound ${ }^{2}$, aidait la musique à se tailler une place dans les collections numériques des bibliothèques.

Les bibliothèques publiques doivent donc maintenant faire face à de nouveaux défis. Comment faciliter l'accès à ces collections ? Comment guider les usagers dans leur exploration? Une façon de répondre à ces questions est d'étudier de quelle façon les gens s'y prennent pour découvrir de nouvelles musiques, puis d'évaluer dans quelle mesure les outils et services proposés par les bibliothèques les soutiennent dans leurs démarches. Cest dans cette optique que fut entrepris le projet de recherche dont nous présentons ici une partie des résultats. Il consistait plus particulièrement à étudier les stratégies auxquelles les jeunes adultes ont recours pour faire des découvertes musicales.

\footnotetext{
http://naxosmusiclibrary.com/

2. http://www.smithsonianglobalsound.org/
} 


\section{Revue de la littérature}

\section{La recherche d'information musicale : un problème complexe}

La recherche d'information musicale se distingue de la recherche d'information textuelle sur plusieurs points. Les études réalisées dans les domaines de la psychologie et de la sociologie de la musique démontrent que le sens de la musique est fortement idiosyncrasique, et donc, fondamentalement subjectif. Selon certains chercheurs, le sens de la musique aurait en fait très peu à voir avec la musique elle-même. Il serait plutôt déterminé par le contexte social dans lequel s'inscrit l'expérience musicale (Bourdieu 1979; Davies 1978 ; Frith 1996). Cette position demeure toutefois relativement marginale. Généralement, on s'entend plutôt pour dire que le sens d'une musique est le résultat d'une relation complexe entre la musique, le paratexte (la pochette d'un disque, les notes dans le livret, etc.) et le contexte social, culturel et biographique dans lequel elle a été entendue (Shepherd et Giles-Davis, 1991). À cela s'ajoute le fait que les caractéristiques intrinsèques de la musique sont complexes : la musique est composée de multiples facettes (hauteur, tempo, timbre, texture, etc.), lesquelles ne sont pas mutuellement exclusives et peuvent dépendre l'une de lautre (Downie, 2003).

La façon dont on détermine le sens de la musique nest pas sans conséquence sur le comportement dans la recherche de musique. Comme l'indiquent Huron et Aarden (2002), « [a]lthough there are many other uses for music, music's preeminent functions are social and psychological. Consequently, we can expect that the most useful retrieval indexes will be those that facilitate searching according to such social and psychological functions ». En d'autres mots, Huron et Aarden nous mettent en garde contre la tentation que nous avons traditionnellement eue d'ignorer les caractéristiques liées aux rôles psychologique et sociologique que joue la musique dans le développement d’outils de repérage en raison du défi que pose leur trop grande subjectivité.

\section{Le comportement des usagers}

Malgré la vigueur de la recherche sur le développement de nouveaux systèmes de repérage et de découverte pour la musique, très peu de chercheurs se sont penchés sur le comportement dans la recherche de musique. Les quelques rares études portant sur ce sujet nous montrent que les systèmes actuels ou en développement ne répondent pas pleinement aux besoins des usagers.

En étudiant le comportement de recherche d'information musicale, des chercheurs ont constaté qu'en dehors des contraintes inhérentes à tout système de repérage, les gens intègrent différents types d'information dans leurs requêtes. Une requête adressée à une personne pourra, par exemple, être composée d'un extrait fredonné d'une mélodie, de métadonnées bibliographiques telles que la période de composition d'une chanson ou un extrait des paroles, d'un exemple d'une chanson similaire à celle que l'on recherche (Downie et Cunningham, 2002). Pourtant, il nexiste aucun outil de repérage permettant aux usagers d'inclure dans une même requête autant de facettes; la très grande majorité des outils offrent un accès, soit au moyen de requêtes textuelles (catalogues de bibliothèques), soit au moyen d'une mélodie fredonnée (systèmes de type "queryby-humming»), soit au moyen d'un ou de plusieurs exemple(s) (systèmes de type "query-by-example» ou de recommandations).

Des études ont également permis de mettre en lumière un besoin pour des notices hautement enrichies (Inskip, Butterworth et MacFarlane, 2008; Lee et Downie, 2004). Dans un sondage, Lee et Downie ont demandé à 427 répondants d'identifier les facettes et critères qu'ils utilisaient pour parcourir ou rechercher de la musique. Ce qui leur a permis de constater quaux métadonnées bibliographiques sajoutaient des données sur les caractéristiques intrinsèques de la musique (tempo, timbre, etc.), des métadonnées associatives (liens entre une musique et un événement, film, publicité, etc.) et des métadonnées relationnelles (relations, notamment de similarité, de collaboration ou d'influence, entre différents artistes).

D'autres chercheurs ont montré qu'il était fréquent dorganiser sa collection personnelle de musique selon la fonction projetée (musique relaxante, pour conduire, pour travailler, etc.) (Cunningham, Jones et Jones, 2004; Vignoli, 2004) plutôt que par genre musical, suggérant qu'il y aurait possiblement un intérêt pour ce type de classification dans les outils de repérage.

Finalement, il est apparu que la recherche de musique était souvent une activité collaborative ou sociale. Il est courant de faire les magasins de disques en groupe (Cunningham, Reeves et Britland, 2003) et on se tourne régulièrement vers les amis ou la famille pour obtenir de l'information sur la musique (Lee et Downie, 2004).

\section{Méthodologie}

Pour étudier la façon dont les jeunes adultes découvrent de nouvelles musiques ou de nouveaux artistes musicaux, des entrevues individuelles semi-structurées ont été réalisées avec 15 adultes francophones, âgés de 18 à 29 ans, habitant la région du Montréal métropolitain. Un guide d'entrevue a été utilisé pour sassurer qu'un certain nombre de thèmes étaient systématiquement abordés de façon à faciliter l'analyse et la comparaison entre les participants. Le guide était composé de questions portant sur la façon dont on découvre de nouvelles musiques dans un contexte de loisirs; il n'incluait donc pas la recherche de musique entreprise dans le but d'accomplir une tâche reliée au travail ou à des études. 
Le recrutement des participants s'est déroulé entre le $1^{\mathrm{er}}$ avril 2006 et le 8 août 2007 dans le hall de l'édifice de Bibliothèque et Archives nationales du Québec. En raison de son emplacement au cœur du centre-ville, sa proximité avec plusieurs institutions d'enseignement et son immense popularité, la Grande Bibliothèque constituait un endroit de choix pour recruter de jeunes adultes aux profils variés. Les participants ont été sélectionnés d'après la stratégie d'échantillonnage à variation maximale (Lincoln et Guba, 1985). Parmi les 15 participants, 10 étaient des hommes et 5 étaient des femmes. $\mathrm{Au}$ moment de l'entrevue, tous détenaient un diplôme détudes secondaires, 13 possédaient un diplôme détudes collégiales ou l'équivalent, et 10 avaient un diplôme universitaire ou étaient inscrits à un programme de niveau universitaire. Parmi ceux qui nétaient plus aux études, 7 travaillaient à temps plein et 3 étaient sans emploi. Six étaient musiciens amateurs. Le groupe était majoritairement composé de grands consommateurs de musique, mais comprenait également des consommateurs plus modérés.

Les entrevues ont été enregistrées puis transcrites. Elles ont été analysées au moyen du logiciel NVivo7 de la compagnie QSR International3, spécifiquement conçu pour l'analyse de données qualitatives.

\section{Résultats et discussion}

En début d'entrevue, les participants étaient invités à parler pendant quelques minutes de leurs habitudes découte de musique afin de les mettre à l'aise et d’obtenir un portrait général de leur consommation de musique dans la vie de tous les jours. La majeure partie de l'entrevue portait sur les sources, formelles et informelles, par lesquelles ils découvrent de nouvelles musiques, plus particulièrement sur la façon dont ils les utilisent et sur ce qui leur plaît ou leur déplaît dans ces sources (Tableau 1). Les participants étaient également appelés

3. http://www.qsrinternational.com/products_nvivo.aspx
L'analyse a révélé que le réseau social, formé typiquement d'amis et de collègues, est de loin la plus importante source de découvertes musicales.

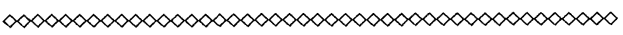

à réfléchir sur ce qui constitue pour eux une expérience agréable ou désagréable de recherche de musique.

Dans cette section, nous présentons une partie des résultats de ce projet de recherche, tout en proposant des pistes de réflexion sur la façon dont on pourrait en tirer profit pour améliorer les services dans les bibliothèques publiques. Des pseudonymes ont été utilisés afin de préserver l'anonymat des participants.

\section{Les personnes comme source d'information : l'entourage et les professionnels}

L'analyse a révélé que le réseau social, formé typiquement d'amis et de collègues, est de loin la plus importante source de découvertes musicales. Bien entendu, la facilité d'accès constitue un avantage, mais ce n'est pas le seul et surtout pas le plus important. En effet, que les suggestions musicales surviennent spontanément au cours d'une conversation ou soient le résultat d'une demande explicite, elles sont considérées comme intéressantes par les participants pour deux raisons :

- "Comme ces personnes connaissent bien mes goûts, leurs recommandations sont personnalisées et ont donc de bonnes chances d'être pertinentes ";

- "Comme je connais ces personnes, je peux juger de la pertinence et de la fiabilité de leurs recommandations ».

Tableau 1 :

Sources d'information les plus utilisées par les participants pour découvrir de nouvelles musiques

\begin{tabular}{|l|c|c|c|}
\hline TYPE DE SOURCES D'INFORMATION & $\begin{array}{c}\text { SOURCE LA PLUS } \\
\text { UTILISÉE }\end{array}$ & $\begin{array}{c}\text { DEUXIÈME SOURCE } \\
\text { LA PLUS UTILISÉE }\end{array}$ & TOTAL \\
\hline Cercle social (amis, collègues, etc.) & 10 & 2 & 12 \\
\hline Ressources Web (Allmusic, MySpace Music, etc.) & 3 & 5 & 8 \\
\hline Radio & 1 & 2 & 2 \\
\hline Magasins de musique & 0 & 0 & 1 \\
\hline Films & 1 & 1 & 1 \\
\hline Bibliothèques & 0 & 1 & 1 \\
\hline Revues/magazines & 0 & 1 & 1 \\
\hline Spectacles/concerts & 0 & & \\
\hline
\end{tabular}




\section{Le rôle de conseiller que jouent les bibliothécaires semble très peu connu des usagers, du moins pour ce qui est des collections musicales.}

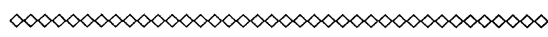

En d'autres mots, c'est l'efficacité quon semble rechercher en faisant appel à son cercle social, une efficacité qui tient au fait que l'information a été filtrée spécifiquement pour le demandeur, par des gens en qui il a confiance et qui ont souvent des goûts similaires aux siens. Ainsi, Karine affirme :

"La personne, c'est un filtre dans le sens que je sais qu'elle a le même âge que moi, elle a les mêmes intérêts que moi, puis elle a peut-être le même profil que moi. [...] C'est un peu comme le shortcut ".

De plus, plusieurs affectionnent les discussions qui accompagnent souvent ces recommandations, car en parlant d'une musique, un ami pourra transmettre sa passion pour celle-ci, indiquer des caractéristiques ou des passages particulièrement intéressants, ou encore fournir des informations sur la vie de lartiste qui permettront de mieux apprécier la musique par la suite. Fannie confirme :

"Parce que des fois, il y a un groupe que je vais avoir découvert par moi-même que j'aurais pas aimé, mais que mon ami va me trouver des arguments par rapport, on dirait, à la musique, puis je vais le réécouter, mais avec un angle nouveau. On dirait qu'ils me font voir une autre vision de la musique».

À l'opposé, les suggestions faites par des disquaires, qu'on ne connait pas et qui ne nous connaissent pas, sont reçues avec beaucoup plus de scepticisme, voire même de méfiance. Selon Benoît : «Si jamais je veux acheter des souliers, je vais pas demander au fabricant de souliers!". Pour ce qui est des quelques participants qui ont dit demander conseil de temps à autre à un disquaire, ils affirment le faire sur une base sélective. On doit pouvoir deviner quel genre de musique ils écoutent, ce qui peut se traduire par l'apparence physique de la personne ("Celle-ci doit "avoir l'air" d'écouter le même genre de musique que moi») ou par l'endroit où elle travaille, le personnel des petites boutiques spécialisées semblant inspirer davantage confiance. Une interaction satisfaisante peut alors mener à une relation de confiance : le disquaire devient une personne ressource dont les recommandations sont hautement estimées.

Les raisons invoquées pour justifier la confiance limitée envers les critiques musicaux sont similaires, comme en témoignent les propos d'Adrien qui explique pourquoi il préfère suivre les recommandations de ses collègues plutôt que celles des critiques :

«Et puis [les critiques], en même temps, ils s'adressent pas juste à toi, en connaissant tes goûts, ils s'adressent à tout le monde donc ça peut pas... c'est pas mal moins fiable, je trouve, comme moyen".

Il est à noter toutefois que le comportement de certains participants entre en contradiction avec leurs propos : d'un côté, on affirme ne pas faire confiance aux critiques et ne pas se laisser influencer, de lautre, on admet les lire régulièrement. Christian affirme: "Je les lis mais je les écoute même pas!". Une seule participante avoue candidement avoir une confiance aveugle dans le jugement d'un critique particulier qui a, selon son point de vue, une grande "crédibilité " alors qu'elle n'a pas «loreille critique» et ne peut donc "pas vraiment savoir ce qui est bon".

Pour ce qui est des bibliothécaires, ils sont tout simplement absents du portrait dressé par les participants. En effet, ceux-ci n'ont jamais demandé des conseils à un bibliothécaire ou à tout autre membre du personnel d'une bibliothèque, d'une part, parce qu'ils ne savaient pas que ça peut faire partie de leur rôle (Danielle : "Est-ce qu'ils sont là pour ça? »), d’autre part, parce qu'encore une fois, ils considèrent qu'il y a peu de chance pour que ce soit fructueux étant donné qu'ils ne connaissent pas leurs goûts propres (Élise: "Il ne me connaît pas du tout! »).

\section{Implications pour les services en bibliothèque publique}

Le rôle de conseiller que jouent les bibliothécaires semble très peu connu des usagers, du moins pour ce qui est des collections musicales. Difficile d'expliquer pourquoi il en est ainsi. Les bibliothécaires ont-ils endossé pleinement ce rôle? A-t-on suffisamment mis de l'avant ce service? Bibliographies sélectives, cercles de lecteurs, expositions thématiques, lectures publiques et étiquettes "coup de cœur" sont courants pour les romans et la poésie, mais qu'en est-il des collections d'enregistrements musicaux ? À notre connaissance, très peu de bibliothèques publiques québécoises présentent des concerts, font venir des musiciens pour une conférence ou réunissent des amateurs de musique pour discuter autour d'un album ou d'un artiste. En ce sens, il apparait donc que les bibliothèques se sont avant tout contentées de collectionner les documents musicaux et de les rendre accessibles à leurs usagers sans vraiment chercher à en faire la promotion ou à animer leurs collections. Une exception intéressante : la Grande Bibliothèque met chaque mois un certain nombre de disques sur poste découte afin que ses usagers puissent faire de nouvelles découvertes.

Notre analyse a également révélé l'importance que joue le réseau social dans les découvertes musicales. 
Nous avons vu que pour faire confiance à un expert, il faut être en mesure de juger à quel type de personne nous avons affaire. Celle-ci doit faire ses preuves et on doit pouvoir déterminer quels sont ses goûts et ses connaissances en musique afin de savoir si ses recommandations sont fiables. Ces conclusions suggèrent encore une fois que créer des groupes d'amateurs de musique pourrait représenter une façon de favoriser léchange d'information musicale entre les usagers tout en permettant à ceux-ci de mieux connaître le bibliothécaire. Des objectifs semblables pourraient être atteints par un bibliothécaire qui maintiendrait un blogue autour de la musique dans lequel il pourrait publier critiques et suggestions et encourager les commentaires des lecteurs. Le catalogue pourrait aussi être un outil utile. Les catalogues de nouvelle génération, encore peu présents dans nos bibliothèques, permettent aux usagers de proposer des critiques et des évaluations, une façon additionnelle de faciliter l'échange d'information entre usagers.

\section{Les ressources Web : MySpace, Allmusic et les sites de recommandations}

Les ressources disponibles gratuitement sur le Web représentent la deuxième plus importante source de découvertes musicales pour les participants à cette étude, le site MySpace étant de loin la plus populaire d'entre elles. Fondé en 2003, MySpace est un site de réseautage social qui a la particularité d'inclure une section musique (MySpace Music) permettant à tout artiste ou groupe musical de créer gratuitement un profil. Le profil d'un artiste consiste en une page Web sur laquelle on peut mettre des photos et de l'information générale (biographie, liste des concerts, influences, etc.), maintenir un blogue et télécharger chansons et vidéos qui peuvent être écoutés ou visionnés en continu par les visiteurs. De plus, de la même façon que les membres réguliers d'un site de réseautage social peuvent avoir des amis, les artistes peuvent avoir leurs amis, lesquels peuvent être d'autres artistes ou encore des membres réguliers. Il s'agit donc pour les artistes d'un moyen de maintenir des liens avec d'autres artistes, mais aussi avec leurs fans.

Étant donné que la liste des amis d'un artiste est publique et fait partie de son espace Web, les visiteurs peuvent la consulter et c'est justement cette caractéristique de MySpace que les participants semblent particulièrement apprécier. En effet, parcourir une telle liste est considéré par plusieurs comme une excellente façon de découvrir de nouveaux artistes, comme si le fait qu'un artiste soit « ami » avec un artiste qu'on estime lui conférait une sorte de légitimité contribuant à la probabilité que ce nouvel artiste nous plaise.

"C'est une bonne façon, [...] dans leurs amis, [de voir] quels groupes ils écoutent, pour trouver quelque chose qui ressemble, les groupes avec lesquels ils font des concerts.... Ah, c'est vraiment, vraiment pratique!». (Nicolas)
Les ressources disponibles gratuitement sur le Web représentent la deuxième plus importante source de découvertes musicales pour les participants à cette étude.

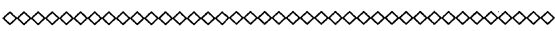

"S'il y a un groupe qui a un ami dans son band c'est souvent parce qu'ils les ont vraiment rencontrés ou ils ont joué avec dans des premières parties ou des affaires comme ça. Je trouve que tu peux plus te fier à ça [qu'aux recommandations des critiques] ». (Jean-Sébastien)

De plus, étant donné que les artistes nont rien à débourser pour avoir un compte sur MySpace, on y retrouve un grand nombre dartistes underground. Il s'agit donc d'une excellente source pour découvrir des artistes que personne d'autre ne connait, ce qui est hautement prisé par les participants.

Parmi les autres sites mentionnés par les participants, on note Allmusic4, un guide dans lequel on trouve de l'information détaillée sur des artistes et des albums de tout genre musical. Pour un artiste donné, on y retrouvera par exemple de l'information factuelle (biographie et discographie), des descripteurs (genre musical, ambiance, instrumentation, période), des artistes associés (influences, "disciples", artistes similaires, collaborateurs) et du contenu éditorial (critiques et évaluations d'albums et de chansons). Le contenu est entièrement développé par des experts. Ce site est apprécié des participants pour son exhaustivité (Nicolas: "C’est une bible!») et pour la fiabilité des informations qu'on y retrouve (Adrien: "C'est pas un Wikipedia, là »). Des informations telles que les collaborateurs et les influences d'un artiste semblent être grandement valorisées, car utiles pour déterminer la probabilité que la musique d'un artiste corresponde à un goût personnel, peut-être davantage que les critiques, considérées comme plus subjectives. De plus, tout comme on parcourt les listes des amis d'un artiste dans MySpace, les adeptes d'Allmusic consultent fréquemment la liste des artistes associés aux artistes qu'ils aiment et naviguent dans le site en cliquant sur les abondants hyperliens qui parsèment les textes, en quête de découvertes.

"Tu peux cliquer sur n'importe quoi, même pendant les critiques, s'ils parlent de quelque chose, c'est highlighté, tu peux aller cliquer si tu sais pas c'est quoi, si tu sais pas c'est qui ». (Nicolas)

4. http://www.allmusic.com/ 


\section{On voit de plus en plus de catalogues grâce auxquels les bibliothécaires peuvent mettre certains documents de l'avant.}

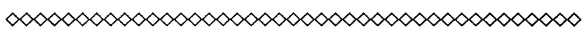

Allmusic est souvent loutil vers lequel on se tourne quand on veut savoir par où commencer pour découvrir un artiste. On consulte la discographie d'un artiste à la recherche des albums ou des chansons les mieux cotés afin de déterminer ce quon écoutera en premier pour se faire une idée. C'est ce que fait Jean-Sébastien :

"S'il y a [...] trop d'albums à écouter puis je veux [...] juste survoler, je prends vraiment ce qui est coté cinq étoiles. Si j'aime pas le cinq étoiles, habituellement...".

D’autres ressources Web ont également été mentionnées, notamment des guides similaires à Allmusic mais portant sur un genre musical particulier. En plus de MySpace, certains participants rapportent avoir fait des découvertes musicales grâce à d'autres sites de réseautage social tels que Facebook et Réseau contact en parcourant la liste des artistes musicaux préférés qui figuraient dans les profils de leurs amis ou contacts. Gabriel en a fait lexpérience :

"Que ce soit sur MSN, Réseau contact, Facebook, peu importe, les gens vont souvent mettre une liste de leurs choix musicaux. Puis quand tu tombes sur quelqu'un qui a des goûts qui sont assez similaires à toi puis il y a des noms que tu ne connais pas, surtout si c'est assez récurrent, tu te dis il faut au moins que j'essaie!"

Pour l'ensemble des ressources Web par lesquelles se fait des découvertes musicales, il est possible de dégager quelques caractéristiques communes contribuant à leur popularité. D'abord, les ressources mentionnées offrent toutes de nombreux liens permettant de naviguer d'un artiste à l'autre, favorisant ainsi la découverte et l'exploration. D'autre part, MySpace, tout comme les sites tels qu'Allmusic, ont en commun de fournir une grande richesse d'information sur les artistes et leur musique. Les raisons pour lesquelles les participants apprécient cet aspect sont multiples. Linformation ainsi acquise permet :

1. de mieux déterminer la probabilité qu’une musique corresponde à ses goûts ;

2. d'enrichir son expérience d'écoute ;

3. de parfaire sa culture musicale.
En fait, accroître sa culture musicale constitue même parfois la motivation principale pour consulter ces sites, surpassant le désir de faire de nouvelles découvertes. Ainsi, en parlant d'Allmusic, Adrien dira : «C'est le genre de site où je vais par habitude, vraiment, pas nécessairement dans le but de télécharger de la musique. J'y vais avant tout pour m'informer".

\section{Implications pour les services en bibliothèque publique}

Il est facile de constater que les catalogues traditionnels offrent peu, comparativement aux ressources Web mentionnées quant aux possibilités de navigation et à la richesse des informations qu'ils proposent sur les artistes et leur musique. L'implantation d'un catalogue de nouvelle génération constituerait une solution intéressante. Ces catalogues permettent d'enrichir les notices bibliographiques d'informations de toutes sortes: critiques, informations biographiques, images numérisées des pochettes d'albums, etc. Ils offrent également une variété de façons d'explorer le catalogue, que ce soit en intégrant différents outils de navigation ou en permettant de proposer des suggestions aux usagers. En effet, on voit de plus en plus de catalogues grâce auxquels les bibliothécaires peuvent mettre certains documents de l'avant, notamment au moyen d'encadrés sur la page d'accueil ou de documents qualifiés de " choix du bibliothécaire " auxquels on donne priorité dans le tri des résultats.

\section{Les lieux physiques : les magasins de disques et les bibliothèques}

Bien que les participants aient affirmé grandement apprécier les ressources Web sur la musique, les magasins de disques et les bibliothèques continuent d'occuper une place de choix dans les sources de découvertes. Les magasins de disques ont particulièrement la cote. Onze participants ont en effet affirmé être des clients réguliers, alors que seulement six déclarent visiter les bibliothèques pour leurs collections d'enregistrements musicaux. Mais qu’on fréquente les magasins de disques ou la section musique d'une bibliothèque publique, les raisons pour lesquelles on le fait sont à peu près les mêmes. On aime $\mathrm{y}$ aller pour prendre son temps: on se promène dans les rayons, sarrêtant au passage pour examiner une section favorite, parcourir le présentoir des nouvelles parutions ou écouter quelques minutes d'un disque sur un poste d'écoute. Martin décrit ses visites chez Archambault de la façon suivante :

"Je me promène puis je regarde [...] Quand on rentre, il y a comme tout le temps les $C D$ les plus vendus ou les plus connus ou qui viennent juste de sortir. Je me mets à les regarder... » 
On apprécie également avoir la possibilité de prendre les albums dans ses mains afin de regarder les pochettes et de parcourir la liste des titres de chansons. De plus, contrairement à la navigation dans les ressources Web, la visite des magasins de disques ou de la section musique d'une bibliothèque peut se faire facilement de façon collaborative, un avantage dont plusieurs participants ont dit tirer parti. On prend en effet plaisir à visiter les disquaires en couple ou entre amis. Pour Alexandre, "[c]'est pratiquement une activité conjugale!». De son côté, Gabriel considère qu'il s'agit d'une excellente occasion de discuter culture entre amis et de "partager des goûts de lecture ou de musique».

La principale critique faite à l'endroit des magasins de musique concerne lécoute de musique sur place dans le but d'évaluer un album en fonction de ses goûts. Il est généralement possible de le faire, mais cela requiert du temps et, surtout, cela peut être intimidant étant donné qu'il faut passer par un membre du personnel. Ainsi, Alexandre explique :

"Tu peux l'écouter si tu veux mais moi, ça me gêne de faire ça, tu sais, d'aller demander: Déballe-moi ça, dude, je vais l'écouter au complet puis je vais te dire non merci! »

Les participants qui fréquentent les bibliothèques publiques pour leurs collections musicales apprécient principalement l'atmosphère calme qui y règne, la diversité des collections (Alexandre dit quon y trouve " tout ce qui est plus difficile à trouver ") et le fait que ce soit gratuit, ce qui les encourage à emprunter des albums d'artistes ou de genres musicaux inconnus. Mais tous nont pas que de bons mots pour les bibliothèques. Certains ne comprennent tout simplement pas l'utilité de se rendre à la bibliothèque pour emprunter des albums qu'on peut télécharger gratuitement sur le Web à partir de chez-soi. Nicolas, au contraire, soppose au principe même du prêt de disques :

" Je trippe pas sur l'idée. C'est comme le paradis des pirates. [...] Ils ont plus besoin d'acheter [l'album], ils se le copient, maintenant tout le monde le fait ".

Finalement, il est à noter que jamais aucun des participants n’a utilisé un catalogue de bibliothèque pour rechercher de la musique, à moins de connaitre d'avance le titre de l'album ou le nom de l'artiste recherché.

\section{Implications pour les services en bibliothèque publique}

Mentionnons d'abord les bons coups des bibliothèques. Lanalyse révèle qu'on aime que les collections denregistrements musicaux soient en accès libre et classés par genre, ce qui permet le bouquinage et incite à la découverte. On note la diversité des collections par rapport à ce quon retrouve dans les magasins
L'analyse révèle qu'on aime que

les collections d'enregistrements

musicaux soient en accès libre et

classés par genre, ce qui permet le

bouquinage et incite à la découverte.

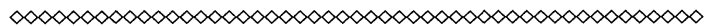

à grande surface. On apprécie la possibilité d'écouter les disques sur place, sans avoir à passer par un membre du personnel et le fait qu'on puisse emprunter gratuitement ces collections5. L'habitude quont déjà les bibliothèques de mettre en valeur certains documents (nouvelles acquisitions, coups de cœur, expositions thématiques, etc.) devrait être étendue aux enregistrements musicaux puisqu'il semble que ce soit une bonne façon pour les usagers de s'exposer de façon plus ou moins arbitraire à la nouveauté.

Notre analyse met également en lumière certains points faibles des bibliothèques publiques. Étant d'abord conçus pour traiter des requêtes précises, dérivées de besoins tout aussi précis, les catalogues traditionnels semblent incapables de soutenir les usagers dans leur exploration de la collection. En effet, ces catalogues sont par exemple bien peu utiles à un usager souhaitant découvrir un artiste dont la musique est «semblable» à celle d'un artiste qu'il connaît et aime déjà. Rappelons que les catalogues de nouvelle génération paraissent mieux outillés pour répondre à ce genre de besoins.

Le fait que plusieurs participants préfèrent télécharger illégalement de la musique sur le Web plutôt que de se déplacer pour emprunter des disques à la bibliothèque suggère que les bases de données permettant découter la musique en continu à distance pourraient répondre de façon plus appropriée à leurs besoins. Cependant, aucun des participants ne connaissait lexistence de ce type de service, et ce malgré le fait que tous étaient abonnés à la Grande Bibliothèque. Cette dernière offrait déjà l'accès à distance à quelques-unes de ces bases de données au moment où les entrevues ont été réalisées. Il est par contre difficile d'expliquer pourquoi il en est ainsi. Peut-être na-t-on pas suffisamment publicisé ce service? Ou peut-être l'information serait-elle davantage connue si les collections disponibles correspondaient plus étroitement à leurs goûts ? On remarque en effet que ces services donnent accès principalement à de la musique classique et à des musiques du monde,

5. Il convient ici de répéter que les participants étaient des usagers de la Grande Bibliothèque. Plusieurs bibliothèques publiques n'offrent pas la possibilité d'écouter de la musique sur place et on en compte un certain nombre qui imposent toujours des frais pour le prêt de disques, ce qui, selon notre analyse, pourrait avoir pour effet de décourager les usagers à prendre des risques et donc à faire des découvertes. 


\section{La recherche de musique est pour plusieurs une activité en soi. Pour être satisfaisante, celle-ci doit être engageante.}

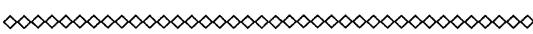

deux genres musicaux très peu populaires parmi les participants.

\section{Commentaires généraux sur les outils de découverte pour la musique}

Il est frappant de constater que, lorsqu'interrogés sur ce qui les motive à faire des recherches sur la musique, les participants ont surtout parlé du plaisir qu'ils en retirent. En fait, qu'on consulte un site Web sur la musique ou qu'on flâne dans un magasin de disques ou une bibliothèque, il s'agit là d'une véritable activité, laquelle peut être satisfaisante en elle-même. Ainsi, on pourra sortir d'une visite chez le disquaire les mains vides, mais avec le sentiment dêtre tout de même comblé. Un participant parle de "hobby" (Jean-Sébastien), une autre compare le temps passé dans un magasin de disque à "aller toute seule un mercredi après-midi manger une pointe de tarte aux pommes". (Laura), alors que pour un autre encore, rechercher de la musique sur le Web s'apparente à jouer à des jeux vidéos :

"À la limite, c'est un substitut aux jeux vidéo.

[...] C'est un aussi bon moyen de se divertir puis

à la limite, c'est moins stressant !»(Adrien)

Il en résulte que plusieurs participants affirment y consacrer beaucoup (certains diront trop) de temps. Quelques-uns avoueront même être parfois si absorbés qu'ils ont du mal à s'arrêter. Christian, qui passe plusieurs heures par jour à chercher de la musique à la bibliothèque et sur le Web, dit: "Des fois, quand jarrive chez nous, jaimerais ça faire autre chose! ». Fannie évoque même la dépendance :

"Je suis un peu accro des fois. Il y a des soirs... je me lève à cinq heures le matin pour aller à l'école, puis il aurait fallu que je sois couchée à huit heures, puis à minuit, je suis encore sur mon ordi!»

On remarque que ce dont on parle vraiment ici, c'est du concept de plaisir dans son sens englobant, lequel se rapproche du concept d' " engagement » dont parlent certains chercheurs. Plus spécifiquement, O'Brien et Toms définissent l'engagement comme " $a$ quality of user experiences with technology that is characterized by challenge, aesthetic and sensory appeal, feedback, novelty interactivity, perceived control and time, awareness, motivation, interest, and affect " (2008). Bien que cette défi- nition s'applique plus spécifiquement à l'interaction entre un utilisateur et une application informatique, il paraît approprié d'étendre son contexte d'utilisation aux systèmes de repérage manuels qu'on trouve dans les bibliothèques et les magasins de disques. Plusieurs des attributs d'une interaction engageante identifiés par O'Brien et Toms ont en effet été mentionnés par les participants pour décrire une expérience de recherche de musique satisfaisante.

\section{Implications pour les services en bibliothèque publique}

La recherche de musique est pour plusieurs une activité en soi. Pour être satisfaisante, celle-ci doit être engageante. On devient alors absorbé dans l'activité et souvent disposé à y consacrer beaucoup de temps. Parcourir les rayons de disques d'une bibliothèque semble avoir la capacité de susciter un sentiment d'engagement, tout comme une visite chez le disquaire ou lexploration de certaines ressources Web sur la musique.

Les catalogues de bibliothèque ne sont pas utilisés par les usagers pour faire des découvertes musicales. En fait, en les examinant, on remarque qu'ils possèdent peu des caractéristiques d'un système engageant. Selon O'Brien et Toms, un système engageant devrait notamment être facile d'utilisation, être esthétiquement agréable et avoir la capacité de maintenir l'intérêt de ses utilisateurs par différents moyens, par exemple, en présentant de nouvelles informations pour susciter la curiosité des utilisateurs ou encore en sollicitant leur participation ou en favorisant l'interaction. Il semble que les catalogues de nouvelle génération possèdent plusieurs de ces caractéristiques et seraient donc plus susceptibles que les catalogues traditionnels déveiller un sentiment d'engagement chez les utilisateurs.

\section{Conclusion}

Cette étude nous a permis de mettre en lumière l'important rôle que jouent amis et collègues dans la découverte de nouvelles musiques chez les jeunes adultes et, à l'inverse, la confiance limitée dont ceux-ci font preuve envers les experts tels que les critiques musicaux, les disquaires et les bibliothécaires. Les ressources Web sur la musique connaissent également une grande popularité : on apprécie la richesse des informations qu'on y trouve sur les artistes et leur musique, de même que les innombrables liens qui relient les artistes entre eux et permettent aux visiteurs de naviguer de multiples façons, en quête de découvertes. Finalement, magasins de disques et bibliothèques continuent dêtre appréciés par cette clientèle qui aime parcourir les rayons et avoir la possibilité de manipuler les albums pour en admirer la pochette ou lire la liste des titres de chansons. Partir à la découverte de nouvelles musiques semble être pour le 
jeune amateur de musique une activité en soi, une façon agréable de se divertir et de passer le temps.

De ces conclusions, nous pouvons tirer quelques suggestions d'amélioration qui pourraient être apportées aux services offerts en bibliothèques publiques afin de mieux guider les jeunes adultes dans leurs découvertes musicales. La principale recommandation serait sans doute d'implanter des catalogues de nouvelle génération, lesquels sont encore très peu présents dans les bibliothèques québécoises. Beaucoup plus ludiques que leurs prédécesseurs, ces catalogues sont engageants et encouragent l'exploration des collections de multiples façons. Lorganisation de cercles d'amateurs de musique et d'autres activités d'animation ou de mise en valeur des collections d'enregistrements musicaux pourraient également favoriser l'échange d'information entre usagers et létablissement d'un lien de confiance avec le bibliothécaire. (1)

\section{Sources consultées}

Bourdieu, Pierre. 1979. La distinction : critique sociale du jugement. Paris : Éditions de minuit.

Cunningham, Sally Jo et al. 2003. An ethnographic study of music information seeking: implications for the design of a music digital library. Proceedings of the third ACM/IEEE-CS joint conference on Digital libraries, $\mathrm{n}^{\circ}$ 5-16.

Cunningham, Sally Jo et al. 2004. Organizing digital music for use : an examination of personal music collections. In ISMIR 2004 : Proceedings of the 5 th International Conference on Music Information Retrieval, sous la dir. de Claudia Lomelí Buyoli et Ramón Loureiro, 447-454. Barcelona, Spain : Universitat Pompeu Fabra.

Davies, John Booth. 1978. The psychology of music. London : Hutchinson.

Downie, J. Stephen. 2003. Music information retrieval. In Annual Review of Information Science and Technology, sous la dir. de Blaise Cronin, 295-340. Medford, NJ : Information Today.

Downie, J. Stephen et Sally Jo Cunningham. 2002. Toward a theory of music information retrieval queries : system design implications. In ISRMI 2002 : Proceedings of the Third International Symposium on Music Information Retrieval, sous la dir. de Michael Fingerhut, 299-300. Paris : Ircam - Centre Pompidou.

Frith, Simon. 1996. Performing rites: on the value of popular music. Cambridge, Massachussetts : Harvard University Press.

Inskip, Charles et al. 2008. A study of the information needs of the users of a folk music library and the implications for the design of a digital library system. Information Processing \& Management $44, \mathrm{n}^{\circ} 2: 647-662$.

Lincoln, Yvonna S. et Egon G. Guba. 1985. Naturalistic inquiry. Beverly Hills, CA : Sage Publications.
La principale recommandation serait sans doute d'implanter des catalogues de nouvelle génération, lesquels sont encore très peu présents dans les bibliothèques québécoises.

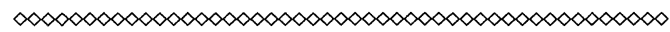

O'Brien, Heather et Elaine G. Toms. 2008. What is user engagement? A conceptual framework for defining user engagement with technology. Journal of the American Society for Information Science and Technology 59, nº $6: 938-955$.

Québec (Province). Ministère de la Culture et des Communications. 200o. Bibliothèques publiques : statistiques 1998.

Québec (Province). Ministère de la Culture, des Communications et de la Condition Féminine. 2008. Bibliothèques Publiques : Statistiques 2006.

Shepherd, John et Jennifer Giles-Davis. 1991. Music, text and subjectivity. In Music as Social Text, sous la dir. de John Shepherd, 174-185. Cambridge : Polity Press.

Vignoli, Fabio. 2004. Digital music interaction concepts : a user study. In ISMIR 2004: Proceedings of the 5th International Conference on Music Information Retrieval, sous la dir. de Claudia Lomelí Buyoli et Ramón Loureiro, 415-420. Barcelona, Spain : Universitat Pompeu Fabra. 